\title{
Model-based sediment classification using single-beam echosounder signals
}

\author{
Mirjam Snellen, Kerstin Siemes, ${ }^{\text {a) }}$ and Dick G. Simons \\ Acoustic Remote Sensing Group, Faculty of Aerospace Engineering, Delft University of Technology, \\ Kluyverweg 1, 2629 HS Delft, The Netherlands
}

(Received 1 September 2010; revised 17 February 2011; accepted 25 February 2011)

\begin{abstract}
Acoustic remote sensing techniques for mapping sediment properties are of interest due to their low costs and high coverage. Model-based approaches directly couple the acoustic signals to sediment properties. Despite the limited coverage of the single-beam echosounder (SBES), it is widely used. Having available model-based SBES classification tools, therefore, is important. Here, two modelbased approaches of different complexity are compared to investigate their practical applicability. The first approach is based on matching the echo envelope. It maximally exploits the information available in the signal but requires complex modeling and optimization. To minimize computational costs, the efficient differential evolution method is used. The second approach reduces the information of the signal to energy only and directly relates this to the reflection coefficient to obtain quantitative information about the sediment parameters. The first approach provides information over a variety of sediment types. In addition to sediment mean grain size, it also provides estimates for the spectral strength and volume scattering parameter. The need to account for all three parameters is demonstrated, justifying computational expenses. In the second approach, the lack of information on these parameters and the limited SBES beamwidth are demonstrated to hamper the conversion of echo energy to reflection coefficient. (C) 2011 Acoustical Society of America.
\end{abstract}

[DOI: $10.1121 / 1.3569718$ ]

PACS number(s): 43.30.Pc [NPC]

Pages: $2878-2888$

\section{INTRODUCTION}

Up-to-date information regarding sea or river floor composition is of high importance for a large number of applications. These include marine geology, marine biology, off-shore construction projects, as well as cable and pipeline route planning. Currently, often samples of the sediments are taken for obtaining the required information. These samples are then analyzed in a laboratory, which is a time-consuming and costly process. An appealing approach, therefore, consists of using acoustic remote sensing techniques for classifying the sediments, employing measurement equipment such as single-beam echosounders (SBES) and multibeam echosounders (MBES), which are already in common use for depth measurements. Whereas SBES acquire a single measurement per ping, the MBES can take up to 500 measurements per ping along a wide swath perpendicular to the direction of navigation. A number of classification approaches for the MBES are presented in literature. ${ }^{1-7}$

Despite the advantages of the MBES, the SBES is still extensively used. Methods that base the sediment classification on SBES measurements are consequently of high interest. Different approaches toward a classification with SBES systems can be found in the literature. ${ }^{8-10}$ In general, these approaches can be divided into two groups, the phenomenological (or empirical) and the model-based (or physical) approaches. In the phenomenological approaches, features such as energy or time spread are determined for the

\footnotetext{
a) Author to whom correspondence should be addressed. Electronic mail: k.siemes@tudelft.nl.
}

received echo signals. These features are known to be indicative for the sediment type. However, independent measurements, such as sediment samples or cores, are needed to link the sediment classes, obtained from signal features, to real sediment properties or sediment type. ${ }^{11,12}$ In contrast, the model-based approaches make use of physical models and determine the seafloor type by maximizing the match between modeled and measured signals or signal features, where seafloor type, or parameters indicative for seafloor type, are input into the model. The advantage of this approach is that, in principle, no independent measurements are needed and the application of a model-based approach directly provides the sediment parameters.

In literature, several approaches toward model-based classification are presented. ${ }^{13-15}$ These approaches mainly differ in the complexity of the sound propagation and sediment interaction accounted for.

In Ref. 13, an approach is proposed that employs a sophisticated model for predicting the SBES echo envelope. This is further described in Ref. 16. The received echoes are modeled as being the result of scattering at the rough sediment interface and at inhomogeneities in the sediment volume. The complexity of the medium accounted for comes at the price of a series of unknowns, requiring efficient optimization methods. For maximizing the agreement between modeled and measured echoes, a combination of different optimization techniques is employed in Ref. 13. Three sediment parameters are searched for, being the sediment mean grain size, the surface roughness, expressed as the spectral strength, and the volume scattering parameter. Inversion of SBES measurements at positions where sediment samples 
were taken show a good agreement between optimized and measured mean grain sizes.

In Ref. 14, the classification is based on the assumption that the SBES echo energy is proportional to the square of the amplitude reflection coefficient. By employing empirical relations between the sediment mean grain size and the reflection coefficient, the echo energy is used to infer the mean grain size for each ping. A comparison of the thus inferred mean grain sizes with the values determined from a sample analysis shows promising results. Increased agreement is obtained by introducing additional complexity in the modeling through the use of a transition layer with a continuous change of sound speed and density.

The fact that model-based approaches allow for obtaining information about the actual sediment parameters, without the need for taking many costly sediment samples, makes them very attractive. In this paper, we therefore investigate the two model-based approaches described above, with the aim to assess their performance for a practical application, which is mapping sediment parameters over areas of interest. The basic principles of the different approaches are taken similar to those described in Refs. 13 and 14. These methods differ in the complexity of the interaction of sound with the sediment accounted for. We investigate the performance of the different approaches, by applying the methods to SBES data taken in an area with a large number of different sediment types. The data used for testing the two approaches were acquired at the Cleaver Bank in the North Sea, The Netherlands.

The first approach is taken similar to Ref. 13 and considers the full echo envelope. As in Ref. 13, the model of the echo envelope comprises both scattering at the water-sediment interface due to roughness and scattering within the sediment body due to inhomogeneities. We reveal the importance of the spectral strength and the volume scattering parameter for obtaining a reliable estimate of the mean grain size. Furthermore, having available information about the spectral strength and volume scattering parameter is of importance, for example, for sonar performance predictions. To compensate for the additional computational effort, when inverting for these two additional parameters, efficient optimization methods are required. For the work presented here, we employ the differential evolution (DE) method, a fast version of the genetic algorithm (GA). ${ }^{17}$ It is demonstrated that this approach, in principle, allows for mapping sediment properties over large areas of interest.

Despite the fact that currently such efficient optimization methods are available, inverting for the full echo envelope is still computational expensive. The actual need of accounting for the full complexity of the backscatter mechanism is investigated by analyzing the approach of Ref. 14, which considers echo energy only. Our analysis reveals the sensitivity of this method to the limited SBES beamwidth and the fact that the actual spectral strength and volume scattering parameter are often not known, which can lead to a non-unique relation between mean grain size and echo energy for SBES systems with small beamwidths.

Section II presents the classification approach which is based on the full echo envelope. In Sec. III, the classification based on the energy of the SBES signal is described. The results of applying these approaches to an SBES data set acquired in the North Sea are discussed in Sec. IV. Finally, conclusions are drawn and recommendations are given in Sec. V.

\section{MODEL-BASED CLASSIFICATION USING THE ECHO ENVELOPE}

The approach taken for the classification is schematized in Fig. 1. Use is made of a physical model that predicts the received SBES return. An optimization algorithm is employed that searches for those input parameters that maximize the agreement between the measured and predicted return. To obtain an overview of the sediment properties over large areas, large numbers of pings need to be inverted for, requiring efficient optimization methods.

The main components of the SBES classification approach are listed below and are described in the following:

(1) the model for predicting the received echo envelopes;

(2) the cost function; and

(3) the optimization method.

\section{A. Modeling the received echo envelope}

For the envelope of the received SBES signal, we can write

$$
y(t)=\int_{A(t)} \sigma_{\mathrm{b}}(\theta) B(\theta) \frac{e^{-4 \alpha r}}{r^{4}} S(r) d A
$$

with $A(t)$ the instantaneously ensonified area that contributes to the sound received at time $t$ and $\sigma_{\mathrm{b}}(\theta)$ the backscattering cross section at the angle of incidence $\theta$, which on its turn depends on $t . B(\theta)$ is the transmit/receive directivity pattern of the transducer and $S(r)$ is the shape of the emitted signal, projected on the footprint to account for the variation of signal amplitude over the footprint. It depends on the slant

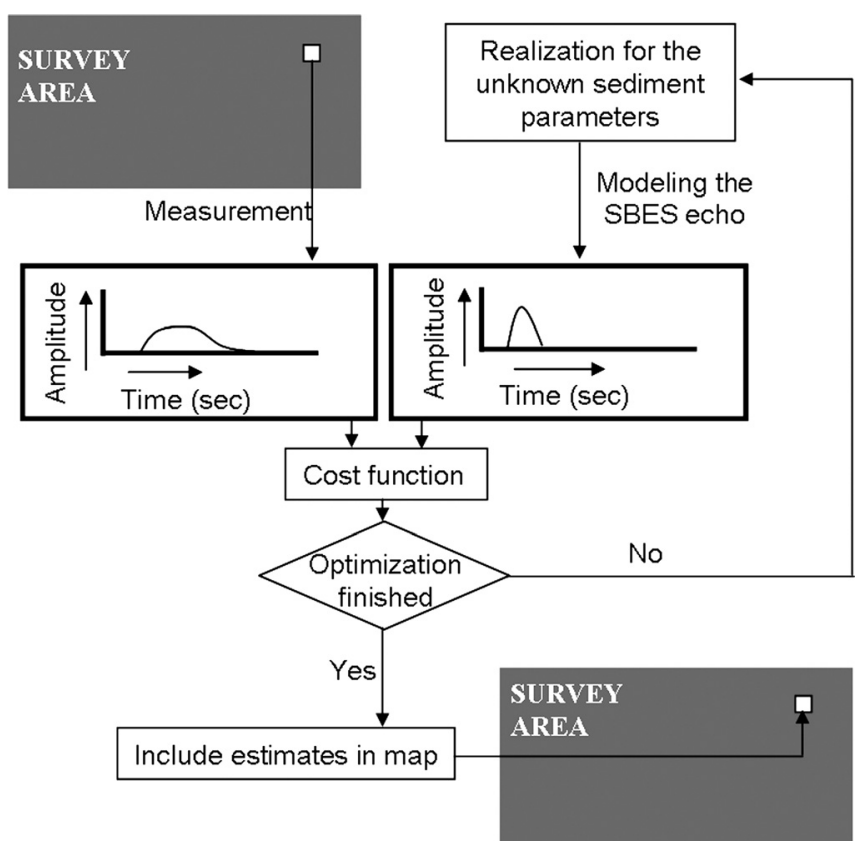

FIG. 1. Schematic of the SBES classification approach. 


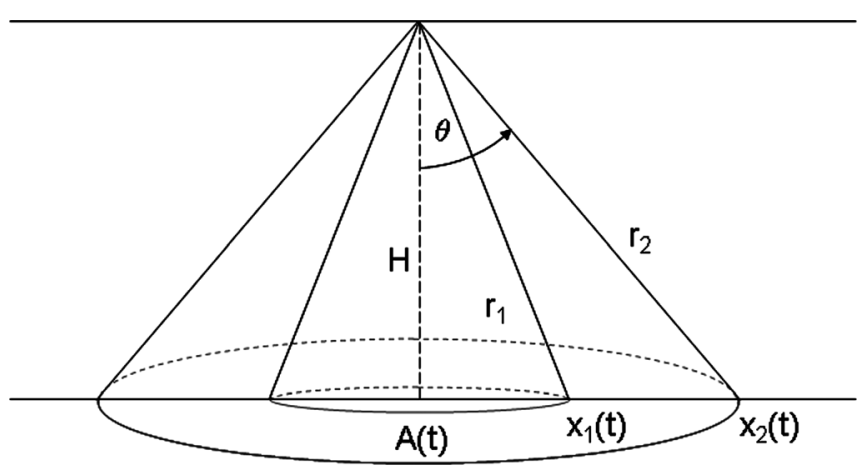

FIG. 2. Schematic of the SBES footprint.

range $r=\sqrt{x^{2}+H^{2}}$, with $x$ the horizontal distance toward the receiver and $H$ the water depth. A schematic is given in Fig. 2. The signal further is corrected for the absorption $\alpha$ in the water column.

Equation (1) can be expanded as follows:

$$
\begin{aligned}
y(t)= & \int_{x_{1}(t)}^{x_{2}(t)} \sigma_{\mathrm{b}}\left(\tan ^{-1}\left(\frac{x}{H}\right)\right) \\
& \times B\left(\tan ^{-1}\left(\frac{x}{H}\right)\right) \frac{e^{-4 \alpha r}}{r^{4}} S\left(\frac{2}{c}\left(r_{2}-r\right)\right) 2 \pi x d x .
\end{aligned}
$$

Here, $x_{1}$ and $x_{2}$ denote the two $x$-values that bound $A=\pi\left(x_{2}^{2}-x_{1}^{2}\right)$, and $r_{2}=\sqrt{x_{2}^{2}+H^{2}}$ is the slant range at $x_{2}$. We have the upper bound

$$
x_{2}(t)=\sqrt{\frac{c^{2} t^{2}}{4}-H^{2}},
$$

with $c$ the speed of sound in the water, which is assumed to be constant. The lower bound $x_{1}$ is dependent on $t$ according to

$$
\begin{aligned}
& x_{1}(t)=0 \text { for } t \leq t_{0}+T, \\
& x_{1}(t)=\sqrt{\left(\frac{c t}{2}-\frac{c T}{2}\right)^{2}-H^{2}} \text { for } t>t_{0}+T,
\end{aligned}
$$

with $t_{0}=2 H / c$ and $T$ the pulse duration.

In general, the transducer characteristics, such as the beam pattern, the signal length, and its shape, are known. This also holds for the data considered in this paper. The water depth can be derived from the measured two-way travel time and the water column sound speed. Consequently, the only unknown in Eq. (2) is the backscattering cross section $\sigma_{\mathrm{b}}(\theta)$. In literature, several expressions for $\sigma_{\mathrm{b}}(\theta)$ are discussed. ${ }^{16,18,19}$ These expressions differ in the level of detail accounted for describing the interaction between sound and sediment. We have considered the considerably detailed and well-established backscattering cross section as presented in Ref. 19, where both the backscatter cross section due to interface roughness scattering $\sigma_{\mathrm{r}}$ and the one due to volume scattering $\sigma_{\mathrm{v}}$ are accounted for

$$
\sigma_{\mathrm{b}}(\theta)=\sigma_{\mathrm{r}}(\theta)+\sigma_{\mathrm{v}}(\theta)
$$

TABLE I. Seafloor parameters and their symbols.

\begin{tabular}{lc}
\hline \hline Seafloor parameter & Symbol \\
\hline $\begin{array}{l}\text { Mean grain size } \\
\text { Ratio of sediment mass density to water } \\
\quad \text { mass density }\end{array}$ & $M_{z}$ \\
Ratio of sediment sound speed to water & $\rho$ \\
$\quad$ sound speed & $v$ \\
$\begin{array}{l}\text { Imaginary to real wave number ratio } \\
\text { Sediment volume scattering cross section } \\
\quad \text { to attenuation coefficient ratio (volume } \\
\quad \text { scattering parameter) }\end{array}$ & $\delta$ \\
$\begin{array}{l}\text { Exponent of the bottom relief spectrum } \\
\text { Strength of the bottom relief spectrum }\left(\mathrm{cm}^{4}\right)\end{array}$ & $\sigma_{2}$ \\
$\quad$ (spectral strength) & $\gamma$ \\
\hline \hline
\end{tabular}

Here, $\sigma_{\mathrm{r}}$ is obtained by appropriate interpolation between the three following approximations:

(1) the Kirchhoff approximation, valid for smooth to moderately rough sediments and angles near nadir;

(2) the composite roughness approximation, valid for smooth to moderately rough sediments and angles away from nadir; and

(3) the large-roughness scattering with a scattering cross section determined from an empirical expression, which is derived for rough sediments like gravel and rock.

For more details, we refer to Ref. 19. The sediment parameters that affect the backscatter cross section are listed in Table I. In Ref. 19, a value of 3.25 for the exponent of the bottom relief spectrum $\gamma$ is found to work well for many types of sediments. Further, empirical expressions are provided in Ref. 19 that relate the density, sound speed, and absorption coefficient (expressed as $\rho, v$, and $\delta$ ), and the parameters affecting the scattering due to surface roughness and volume inhomogeneities (expressed as $w_{2}$ and $\sigma_{2}$ ) to a single parameter, being the mean grain size. The mean grain size is often expressed in $\phi$ units as

$$
M_{z}=-\log _{2} d
$$

where $d$ denotes the mean grain size in millimeters. For mean grain sizes of $-1 \phi$ to $9 \phi$, the empirical parameter values of the above mentioned parameters are listed in Table II.

TABLE II. Empirical values of the model parameters.

\begin{tabular}{lccccc}
\hline \hline$M_{z}(\phi)$ & $\rho(-)$ & $v(-)$ & $\delta(-)$ & $\sigma_{2}(-)$ & $w_{2}\left(\mathrm{~cm}^{4}\right)$ \\
\hline-1.0 & 2.492 & 1.337 & 0.0171 & 0.002 & 0.0129 \\
0.0 & 2.314 & 1.278 & 0.0163 & 0.002 & 0.0086 \\
1.0 & 2.151 & 1.224 & 0.0165 & 0.002 & 0.0056 \\
2.0 & 1.615 & 1.140 & 0.0161 & 0.002 & 0.0035 \\
3.0 & 1.339 & 1.080 & 0.0173 & 0.002 & 0.0021 \\
4.0 & 1.223 & 1.036 & 0.0202 & 0.002 & 0.0011 \\
5.0 & 1.169 & 1.000 & 0.0126 & 0.002 & 0.0005 \\
6.0 & 1.149 & 0.987 & 0.0039 & 0.001 & 0.0005 \\
7.0 & 1.147 & 0.985 & 0.0024 & 0.001 & 0.0005 \\
8.0 & 1.146 & 0.982 & 0.0016 & 0.001 & 0.0005 \\
9.0 & 1.145 & 0.980 & 0.0015 & 0.001 & 0.0005 \\
\hline \hline
\end{tabular}


By employing these empirical expressions, the search for these parameters can be reduced to a search for the mean grain size only. However, the spectral strength $w_{2}$ and the volume scattering parameter $\sigma_{2}$ are known to possibly deviate significantly from default values obtained from expressions relating them to $M_{z}$ and are, therefore, included in the search. To assess the need to indeed account for all three unknowns, we also present inversions where only the mean grain size is optimized and the empirical expressions are used to assign values to the spectral strength and volume scattering parameter, based on the mean grain size.

\section{B. The cost function and the optimization method}

In the model-based approach, parameters of the seafloor are derived by maximizing the match between the measured signal and the modeled signal. As a measure for the agreement between modeled echo signal [conform Eq. (1)] and measured echo signal, the following cost function $E$ has been defined

$$
E=\frac{1}{\sum_{k}\left(y_{\text {meas }}^{2}\left(t_{k}\right)+y_{\text {mod }}^{2}\left(t_{k}\right)\right)} \sum_{k}\left[y_{\text {meas }}\left(t_{k}\right)-y_{\text {mod }}\left(t_{k}\right)\right]^{2} .
$$

Here, $y_{\text {meas }}$ and $y_{\text {mod }}$ denote the measured and modeled echo envelope, respectively, and $k$ is the number of time samples considered, with $t_{k}$ denoting the corresponding times.

In order to obtain an overview of the sediment distribution over an area, a large number of SBES measurements needs to be inverted for. This requires the use of efficient optimization methods for minimizing $E$. In Ref. 20, DE, which is a fast version of a GA, was demonstrated to be an efficient global optimization method. Therefore, we have employed this method for minimizing $E$. A description of $\mathrm{DE}$ is given in Ref. 17. Based on a synthetic study ${ }^{21}$ and preliminary inversions, DE settings were selected such that 2400 forward calculations were applied.

\section{MODEL-BASED CLASSIFICATION USING THE ECHO ENERGY}

Since information of the full echo envelope is not always available and employing it for the inversion of $M_{z}$ has proven to be computational extensive (Sec. II), the merit of alternatively employing the echo energy only for classifying the sediment is analyzed in the following.

From existing phenomenological classification approaches, it is well known that the energy of the received signal allows for discriminating sediment types. ${ }^{11,12}$ This knowledge is fully exploited in Ref. 14, where the received echo energy is assumed to be directly proportional to the square of the amplitude reflection coefficient at normal incidence. Empirical relations then relate the reflection coefficient to the mean grain size.

Assuming that all received energy is the result of reflection at the water-sediment interface, the amplitude reflection coefficient can be determined from the SBES echo energy as

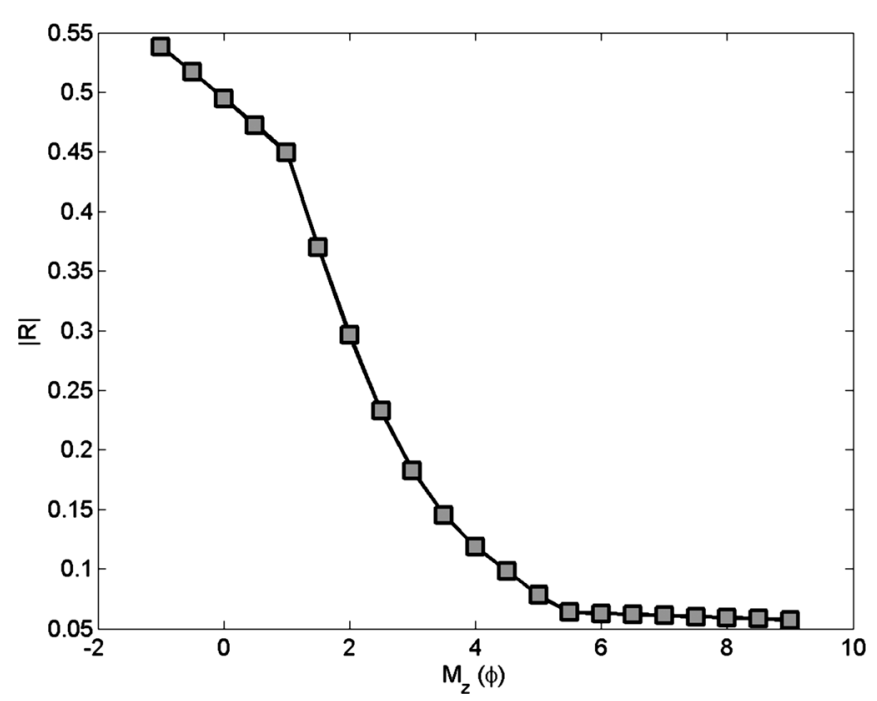

FIG. 3. Theoretical relation between sediment mean grain size and reflection coefficient, obtained from Eq. (9).

$$
R=\frac{1}{\sqrt{E_{\mathrm{TX}}}} \frac{2 H \sqrt{E_{\mathrm{RX}}}}{e^{-2 \alpha H}}
$$

with $R$ the amplitude reflection coefficient, $H$ the water depth, $E_{\mathrm{TX}}$ the energy of the transmitted pulse, $E_{\mathrm{RX}}$ the energy of the received pulse, and $\alpha$ the absorption coefficient in the water.

Based on the empirical relations in Ref. 19, the relation between $M_{z}$ and the reflection coefficient, $R$, is calculated based on the sediment-water ratio of mass density, $\rho$, and the sediment-water ratio of sound speed, $v$, under the assumption of normal incidence.

$$
R\left(M_{z}\right)=\frac{\rho\left(M_{z}\right) \cdot v\left(M_{z}\right)-1}{\rho\left(M_{z}\right) \cdot v\left(M_{z}\right)+1} .
$$

The resulting reflection coefficient is presented in Fig. 3. Whereas Eq. (9) potentially allows for estimating the mean grain size from the received echo energy, Fig. 3 illustrates that $R$ is not sensitive to mean grain sizes larger than $5.5 \phi$.

\section{APPLICATION OF THE METHODS TO SBES DATA}

\section{A. Description of the data set}

To assess the performance of the classification methods, described in Secs. II and III, for real data, SBES data acquired from the North Sea in November 2004 are considered. The area where the data were taken is located close to the Cleaver Bank and Botney Cut, north-west of the Netherlands. The area is of interest due to its variability in sediment types, which was already indicated in Ref. 22. Water depths in the area vary between 30 and 60 m, as illustrated in Fig. 4 .

A dense pattern of east-west tracks was sailed, while taking the SBES measurements. The measurements considered here were taken by a $38 \mathrm{kHz}$ Kongsberg EA600 SBES system (Kongsberg Maritime, Kongsberg, Norway). This echosounder has a beamwidth of $9.6^{\circ}$ and a pulse length of $256 \mu$ s. Ping rates typically are $5 \mathrm{~Hz}$. 


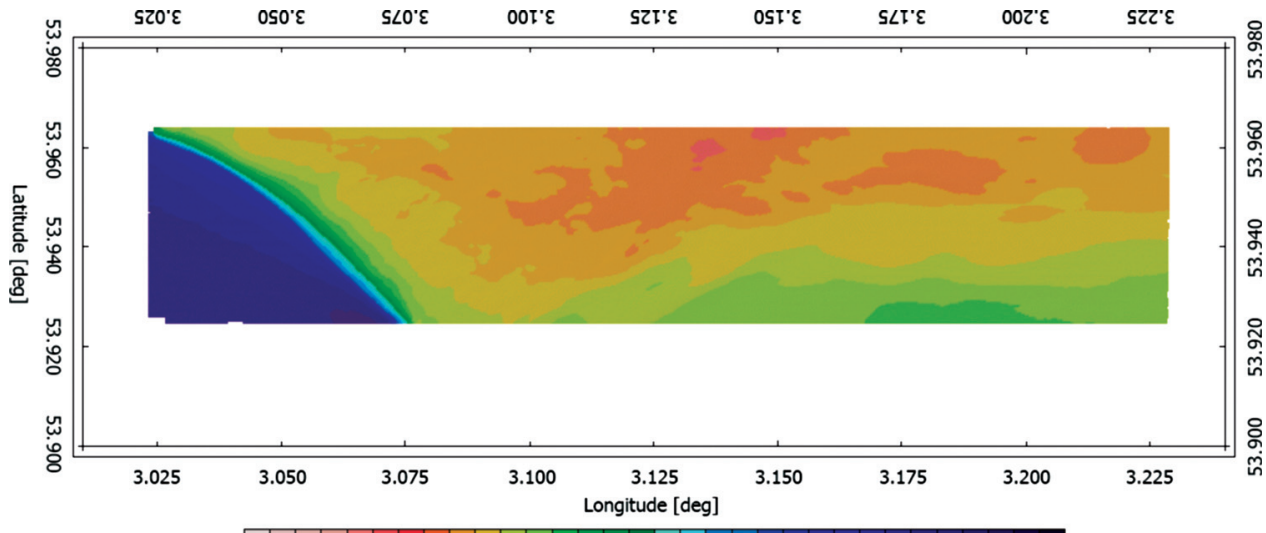

FIG. 4. Water depth in the trial area.

$\begin{array}{lllllllllllllllll}25.0 & 27.5 & 30.0 & 32.5 & 35.0 & 37.5 & 40.0 & 42.5 & 45.0 & 47.5 & 50.0 & 52.5 & 55.0 & 57.5 & 60.0 & 62.5 & 65.0\end{array}$ [m]

The individual SBES returns indicate a clear ping-toping variability, which is due to the stochastic nature of the backscatter process, motions of the ship, and presence of bottom features. To constrain these effects at least partly and instead capture the effects of seafloor type on the received echoes, a series of sequential pings is averaged. Here, the averaging was carried out over 50 sequential pings, which cover a distance of approximately $50 \mathrm{~m}$. This value was chosen as a compromise between the aim to average out the above described effects and still keeping an acceptable spatial resolution.

Information about the sediment composition is available from an earlier sea trial in November 2000. During this trial, a series of bottom grab samples was taken. The laboratory

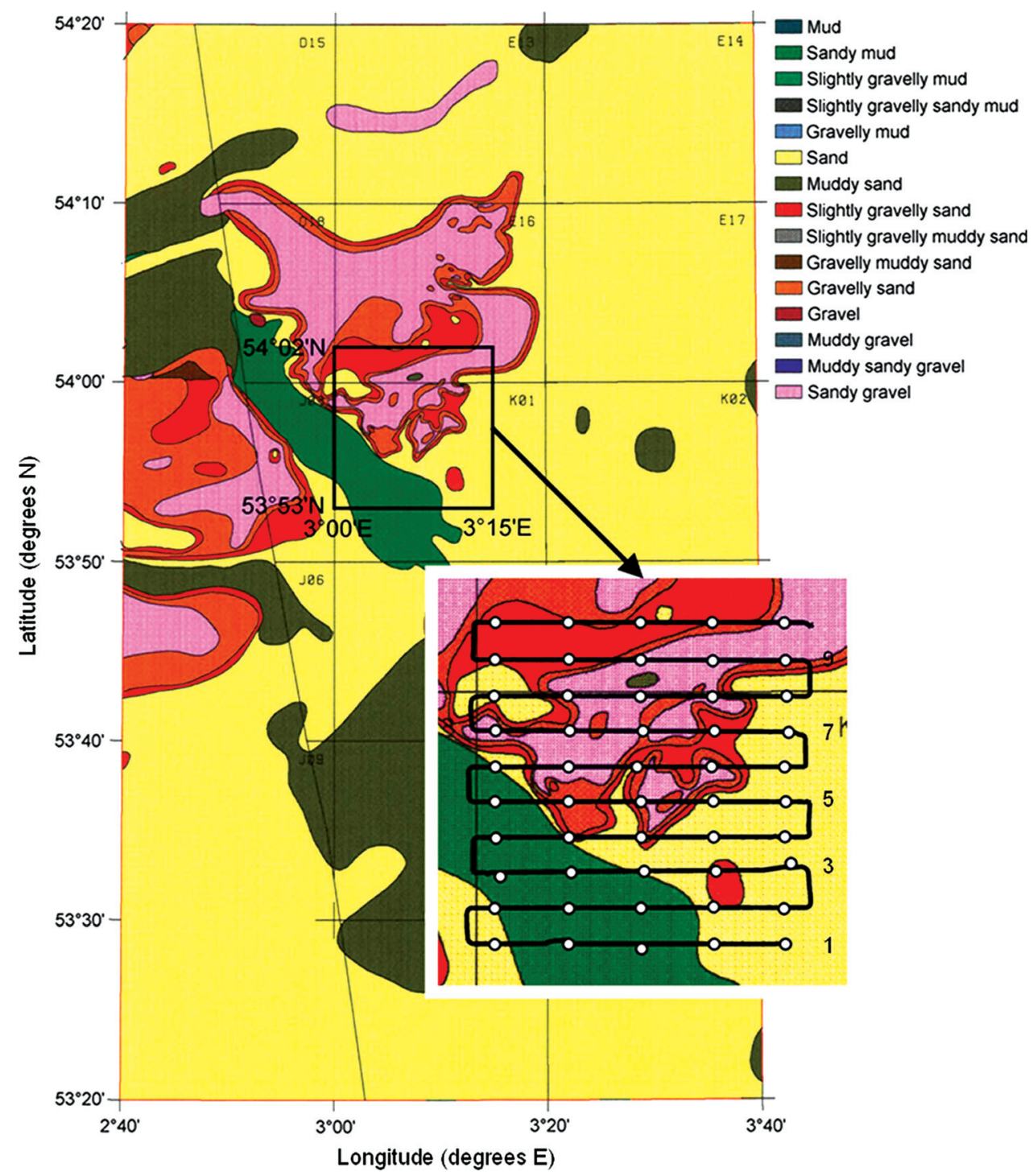

FIG. 5. Overview of the sediment Folk classes in the trial area, taken from Ref. 21. 
TABLE III. Relation of Folk classes to mean grain size.

\begin{tabular}{lc}
\hline \hline Folk class & $M_{z}(\varphi)$ \\
\hline Sandy gravel & -1 \\
Muddy sandy gravel & 0 \\
Gravely sand & 0.5 \\
Slightly gravelly sand (also denoted as gravelly & 1 \\
$\quad$ muddy sand in Ref. 19) & 1.5 \\
Sand (also denoted as medium sand in Ref. 19) & 3 \\
Muddy sand & 5 \\
Slightly gravelly sandy mud (also denoted as & \\
$\quad$ sandy silt (or gravelly mud) in Ref. 19) & 6 \\
Sandy mud & \\
\hline \hline
\end{tabular}

analysis of the sediment samples comprised the following steps. First, the samples were dried. Then, the samples were sieved with a mesh of $2 \mathrm{~mm}$, thereby separating the gravel and shells from the sand and mud. Both, the gravel and shell weight percentage were subsequently determined. The precise grain size distribution of the sediment samples (after the gravel was removed) was then determined by optical microscopy. From these, values for the $10 \%, 50 \%$, and $90 \%$ threshold of the grain sizes were determined. Additionally, the grab samples were labeled according to the Folk scheme based on the percentages of mud, sand, and gravel. ${ }^{23}$ An overview of the Folk classes in the trial area is given in Fig. 5.

Mean grain sizes have been assigned to the grab samples by relating them to Folk classes, as shown in Table III. This relation is based on Ref. 19. No use was made of the also available values for $10 \%, 50 \%$, and $90 \%$ thresholds of the grain sizes, since these are based on the sand fraction only, thereby not accounting for the presence of gravel.

In total, 20 grab samples were taken in the area under investigation, comprising legs 3-6 in Fig. 5. However, distances between the grab positions and the SBES tracks are at least $50 \mathrm{~m}$.

Further, small-scale variations in the sediment distribution in the 4-yr time span between the sediment sampling (2000) and the acoustic measurements (2004) are expected. However, we expect these small-scale variations to have little influence on the overall sediment distribution in the area.
Therefore, we assume that the sediment samples can still be used for comparison with the acoustic classification results.

\section{B. Classification based on employing the full echo envelope}

The averaged SBES pings were used in the inversion process that accounts for the full echo envelope, as schematized in Fig. 1. In total, about 16000 averaged pings have been inverted, covering the entire trial area. The parameters inverted for are the mean grain size $M_{z}$, the spectral strength $w_{2}$, and the volume scattering parameter $\sigma_{2}$. The resulting estimates for mean grain size are shown in Fig. 6. In addition, mean grain sizes as determined from the Folk classes of the grab samples are included in the plot.

The spatial behavior indicated by the Folk classes, showing coarser sediments in the center part of the area, is in agreement with that of the inverted mean grain size. For the 60-m deep trench in the south-western part of the area, inverted $M_{z}$ values indicate the presence of fine sediments $(\geq 4 \phi)$. This is expected due to the, in general, lower currents in the deeper area and is in agreement with the $M_{z}$ values obtained from the grab samples. In the remainder of the area, both sets of $M_{z}$ values are lower, indicating coarser sediments. Here, however, they agree to a lesser extent. Whereas, the grab samples indicate only a minor variation in composition for the south-eastern area, resulting in the same mean grain size of typically $1.5 \phi$, the inversions reveal consistent regions with different mean grain size values, spanning a range of about $4 \phi$. The ability to identify these structures from the grab samples is at least partly hampered by the small number of grab samples.

A more detailed comparison, for example, aiming at establishing a relation between the estimated $M_{z}$ and all other parameters of the samples (shell percentage; gravel percentage; and values for the $10 \%, 50 \%$, and $90 \%$ threshold for grain sizes) is not feasible due to the limited number of grab samples. In addition, the grab samples are located not exactly along the acoustic tracks. Distances between grab samples and acoustic samples are at least $50 \mathrm{~m}$, whereas the acoustic classification indicates variability on typically these scales.

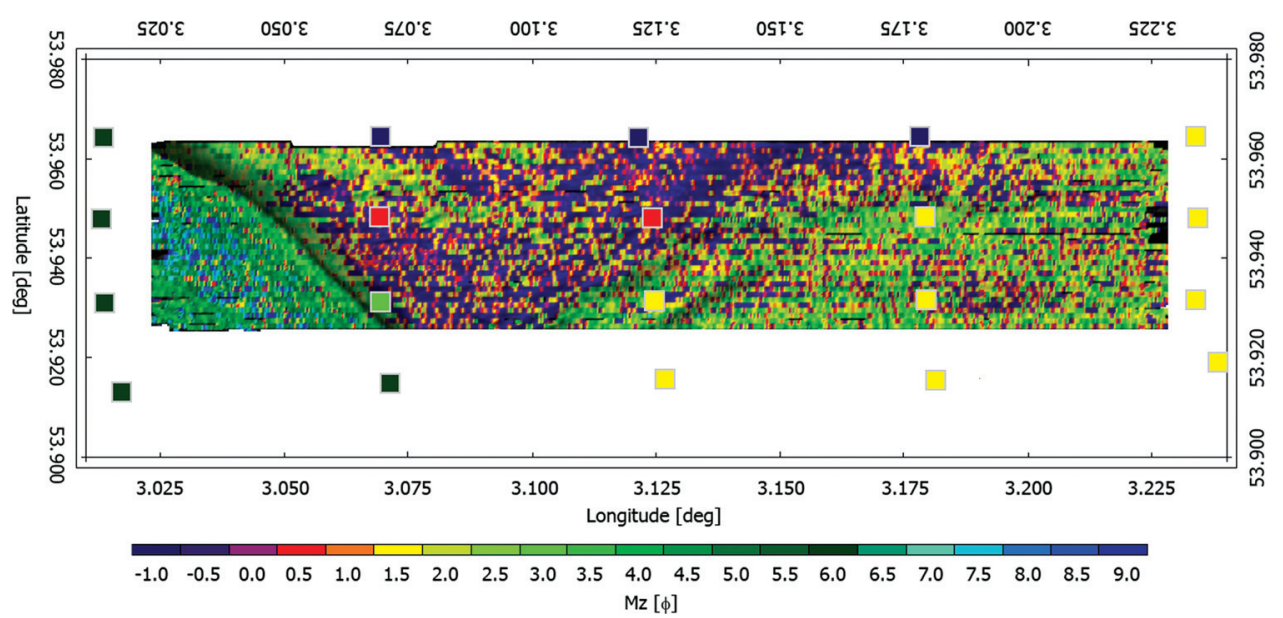

FIG. 6. Map of inverted mean grain size. Also shown is the measured mean grain size as obtained from the grab samples as the colored squares. For the grab samples the Folk classes are displayed, which are related to $M_{z}$ according to Table III. 


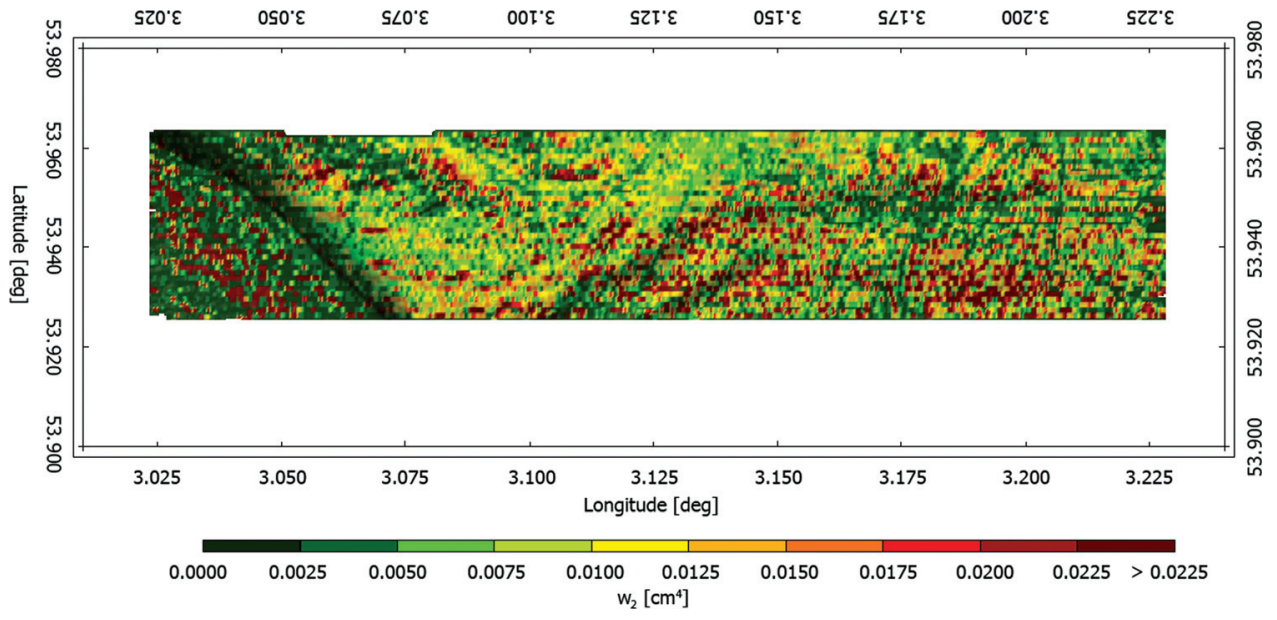

FIG. 7. Map of the inverted spectral strength $w_{2}$.

Figures 7 and 8 present the estimates for the spectral strength and the volume scattering parameter, respectively. In general, these two estimated parameters reveal a similar spatial pattern as $M_{z}$, dividing the area into three distinct regions.

In Fig. 7, clear lines are visible in the eastern part. In Ref. 14, it is hypothesized that these lines are caused by fishing gear dragged over the seafloor. As can be observed from the inversion results, these plough marks affect the spectral strength, but not the mean grain size and volume scattering parameter, as expected.

Figure 8 indicates higher values for the volume scattering parameter for clay $\left(0<\sigma_{2}<0.0075\right)$ than for fine sand $\left(0.005<\sigma_{2}<0.0175\right)$. As expected from Ref. 19, the highest values are found for the coarsest sediments that include gravel $\left(\sigma_{2}>0.00175\right)$.

For $w_{2}$ and $\sigma_{2}$, no independent measurements are available for validation purposes. In order to assess the sensitivity of the inversion to these two parameters, we have also carried out inversions where only $M_{z}$ is estimated and empirical relations (Table II) are employed that express the spectral strength and volume scattering parameter as a function of mean grain size. The results are presented in Fig. 9. Comparing these to the results in Fig. 6, where $M_{z}$ is determined from the inversion of all three parameters, it can be seen that both figures indicate the same distribution of the coarse and fine sediments. Inverting for $M_{z}$ only, however, features higher $M_{z}$ estimates, thus finer sediment grains, resulting in a decreased correspondence with the mean grain size estimates from the grab samples. In contrast, correspondence is somewhat enhanced in the center part of the coarse grained area. Furthermore, the presence of plough marks is now revealed in the map as lines with lower $M_{z}$ values.

The parameters $w_{2}$ and $\sigma_{2}$ are known to often deviate significantly from the values as predicted by the empirical relations; see Table II as taken from Ref. 19. For the results shown in Fig. 9, the deviation of $w_{2}$ and $\sigma_{2}$ from the empirical expressions contributes to mean grain sizes that differ from their real values. By not relying on these empirical relations, more realistic values for $M_{z}$ will consequently be obtained and, therefore, Fig. 6 should represent the reality better than Fig. 9. Furthermore, the obtained estimates for the spectral strength and volume scattering parameter provide additional information about the sediment characteristics.

Computation times, however, are significant for the three-parameter model. Using a standard personal computer (PC) and MATLAB as the programming language, inverting a single averaged ping requires several minutes computation time. Despite these computation times for the three-parameter

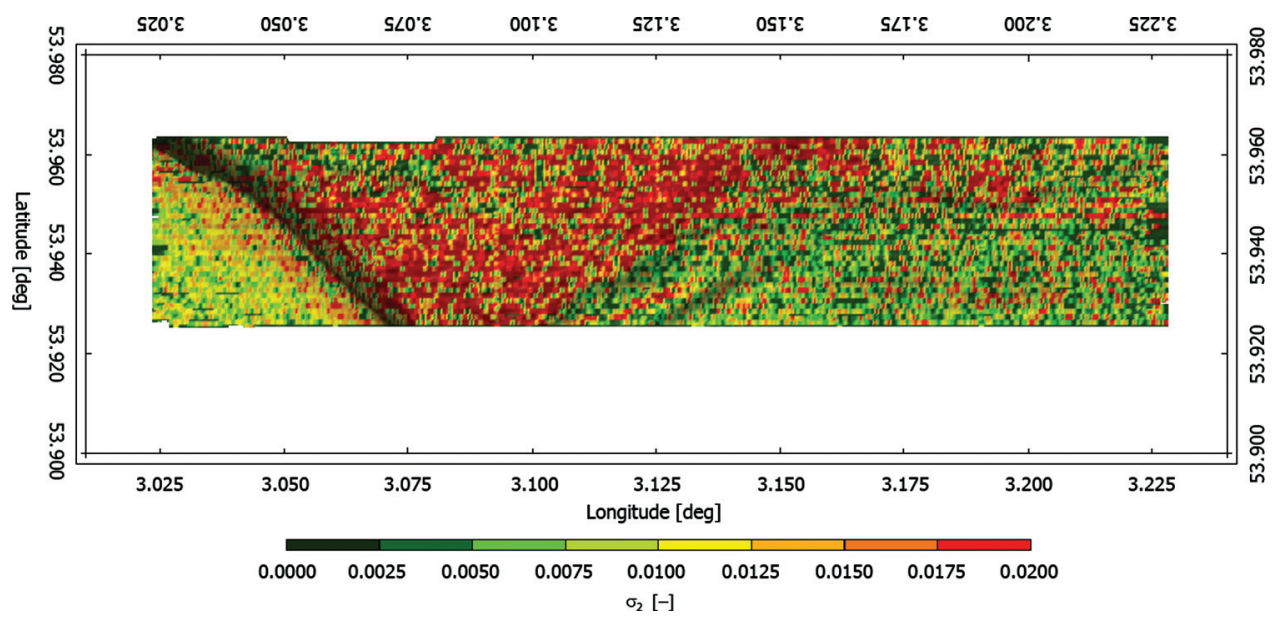

FIG. 8. Map of the inverted volume scattering parameter $\sigma_{2}$ 


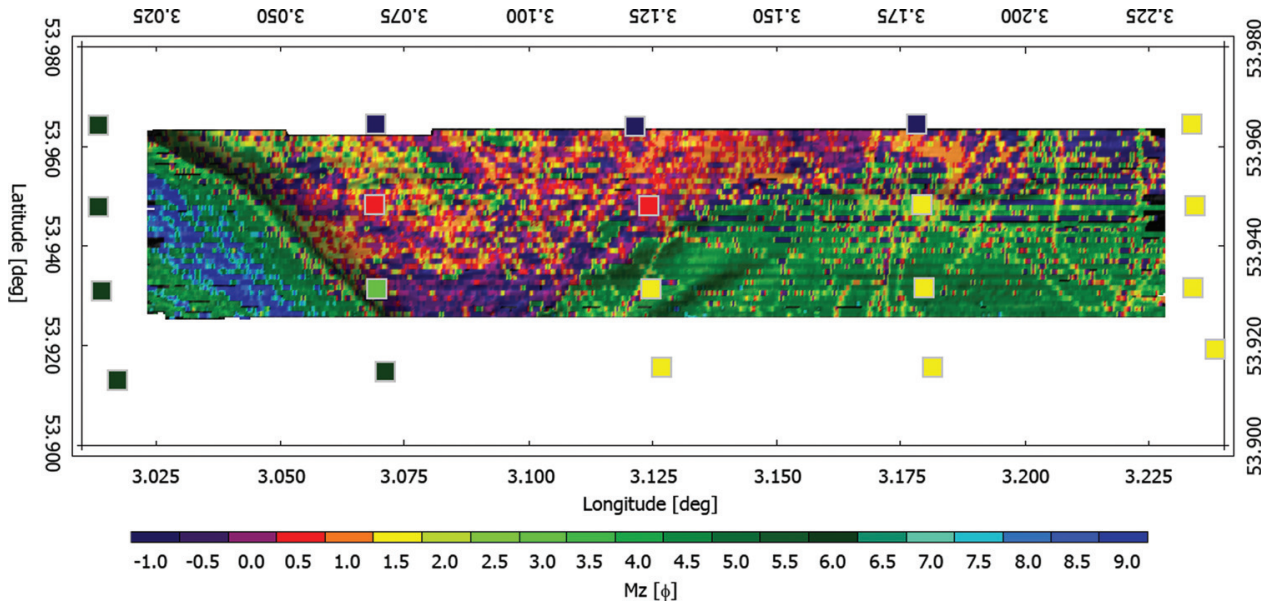

FIG. 9. Map of the mean grain size obtained from optimization where only $M_{z}$ is searched for.

model, off-line mapping is feasible as shown by Figs. 6-8. Beside its possibility of classifying and mapping sea bottom sediments, this approach can also contribute to a gain of insight into the scattering process, when applied to a large number of different data sets. This would, consequently, allow for improving the modeling capabilities.

\section{Classification based on the reflection coefficient}

The second classification approach under consideration estimates the mean grain size from the SBES reflection coefficient, which can be obtained from Eq. (8), by using Eq. (9). Due to the resulting direct coupling between echo energy and sediment mean grain size, no optimizations are needed, making this a tempting approach.

Here, the same averaged SBES signals are considered as used for the inversions employing the full echo envelope (Sec. IV B). Figure 10 presents the reflection coefficients estimated from these signals, whose values lie in the range from 0.05 to 0.3. According to the theoretical relation in Fig. 3 , these values of the reflection coefficient can be related to $M_{z}$ values larger than $2 \phi$. However, from the analysis of the samples and also from the inversion results for the full echo envelope, see Fig. 6, lower $M_{z}$ values are known to occur in the area.
To investigate possible causes of this effect, we have used Eq. (2) to predict the envelope of the SBES returns for a series of mean grain size values. For the grain sizes considered, all other sediment parameters of the model, including also $w_{2}$ and $\sigma_{2}$, are calculated according to the empirical relations of Ref. 19, as given in Table II. For each of the predicted signals, the echo energy is determined. Then, Eq. (8) is used to obtain an estimate for the reflection coefficient based on this echo energy. The left plot in Fig. 11 shows the results for an SBES with a beamwidth of $9.6^{\circ}$, as considered throughout this paper.

Clearly, the reflection coefficient estimated from the simulated signal is much lower for the coarse grains than expected. The main reason for this deviation is the limited beamwidth of the transducer, which strongly reduces the amount of sound that impinges upon the SBES at angles away from normal incidence. This also causes non-uniqueness in the conversion of energy to mean grain size, hampering the mapping of the mean grain sizes based on the reflection coefficient. Using an SBES with a larger beamwidth will reduce these effects. ${ }^{24}$ This is demonstrated in the right plot of Fig. 11, where the same procedure as above is followed for an SBES with an increased beamwidth of $30^{\circ}$. However, the contribution of the volume scattering now

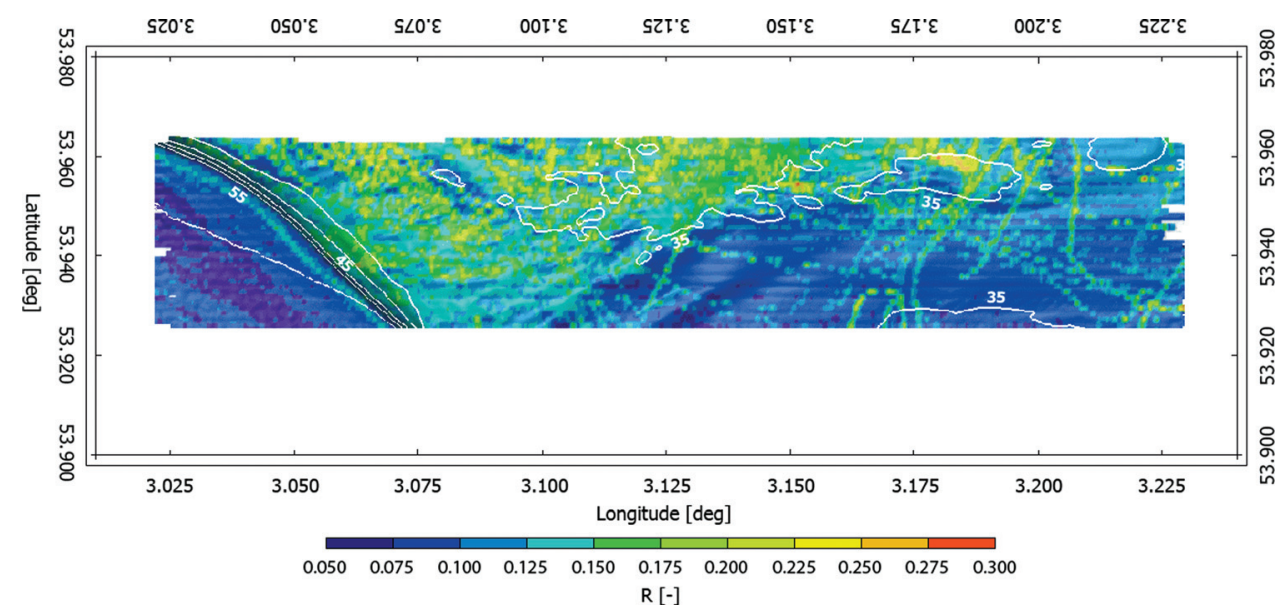

FIG. 10. Map of the reflection coefficient as determined from the averaged SBES returns. The white lines indicate equal bathymetry contours. 

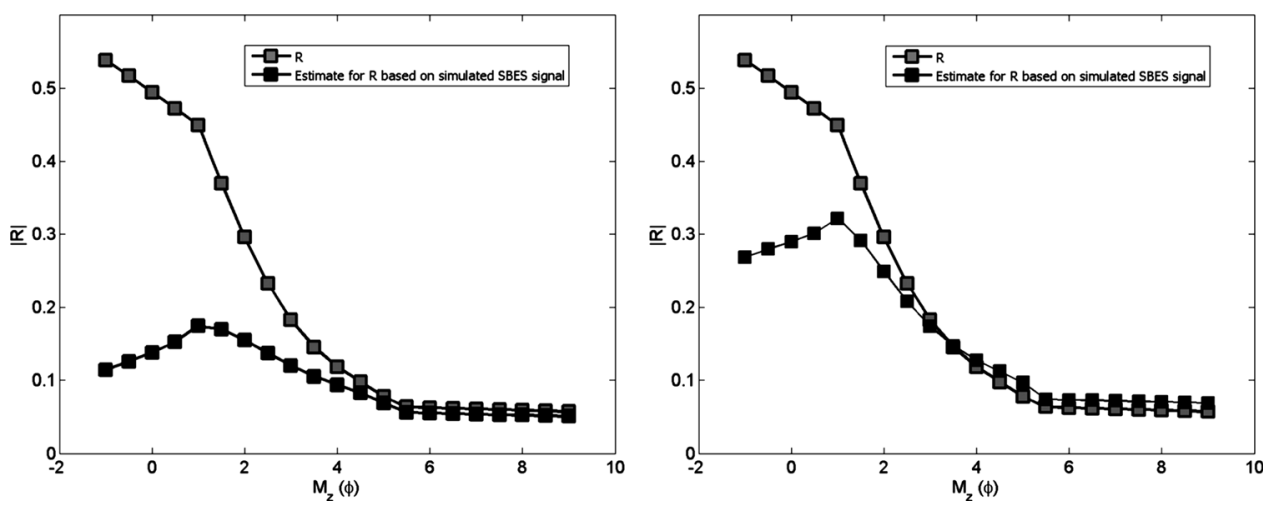

FIG. 11. Theoretical relation between the mean grain size and the reflection coefficient, one reproduced from Fig. 3 (gray squares) and the other obtained from the energy of the simulated SBES signals (black squares) for SBESs with a beamwidth of $9.6^{\circ}$ (left) and $30^{\circ}$ (right).

results in a reflection coefficient that slightly exceeds the theoretical curve for the fine grained sediments.

Still, for the SBES with a standard beamwidth, as the $9.6^{\circ}$ considered here, regions with different sediment types are revealed by the echo energy.

An additional effect, hampering the coupling of the detected regions of the estimated reflection coefficient to mean grain size, is the uncertainty in the spectral strength and volume scattering parameter, as shown in the following. Again, the only parameter searched for is the mean grain size, whereas all other parameters affecting the interaction of sound with the seafloor are taken according to the assumed empirical relations (Table II). As mentioned before, these relations are known to hold well for the sediment sound speed, density, and attenuation, but less for the spectral strength and volume scattering parameter. ${ }^{19}$ While, in general, these parameters are not known, here, the inversions of the full echo envelope (Sec. IV B) have provided estimates for $w_{2}$ and $\sigma_{2}$ for each averaged signal, in addition to the estimates for $M_{z}$. This allows for assessing the influence of deviations in $w_{2}$ and $\sigma_{2}$ from their empirical values on the estimates of the reflection coefficient.

For this purpose, the measured values of the reflection coefficient are plotted in Fig. 12 versus the available estimates of $M_{z}$, obtained from the inversions using the full echo envelope, as mapped in Fig. 6. Additionally, the graph of Fig. 11, showing the theoretical relationship between the reflection coefficient and mean grain size for an SBES with $9.6^{\circ}$ beamwidth, is added for comparison. In case the empirical relations between the mean grain size and the spectral strength and volume scattering parameter hold, the measured reflection coefficient versus inverted mean grain size would repeat the theoretical relation. However, the values of the reflection coefficient show a spread around the theoretical values, further adding to the non-uniqueness of their relation. Maximum measured values of $R$ can be twice as large as the simulated values. An exception holds for mean grain sizes between $2 \phi$ and $5 \phi$, where the upper bound of the reflection coefficient is more restricted (only 1.5 times of the simulated value). The lower bound of $R$, on the other hand, is approximately 0.05 throughout the entire range of mean grain sizes, disallowing an allocation of mean grain sizes at these small reflection coefficients.

We hypothesize that a major part of the spread is caused by deviations of the spectral strength and volume scattering parameter from their empirical values, in addition to effects of a possibly not fully converged optimization and a possible imperfectness of the model. In Fig. 12, the spectral strength is indicated by the color. For the relation between the reflection coefficient and the inverted mean grain size, the colors represent the inverted spectral strength, whereas for the theoretical relation, spectral strength values as obtained from the empirical expressions are shown. The latter indicate an increasing spectral strength with decreasing $M_{z}$. Despite the large spread, the same trend is visible from the estimates for the spectral strength obtained from the inversion.

Considering the variation of the spectral strength per mean grain size, the plot indicates a decreasing reflection coefficient as a function of increasing spectral strength. This is expected, since increased roughness results in an increased amount of scattering away from normal incidence and consequently a lower signal strength received by the SBES. The spread in $R$ can thus, at least partly, be related to a spread in $w_{2}$. The largest deviation of the spectral strengths from their empirical values is found for coarse sediments. On the other hand, deviations in the volume scattering parameter from their empirical values (not shown here) mainly contribute to the softer sediments. The values for the volume scattering

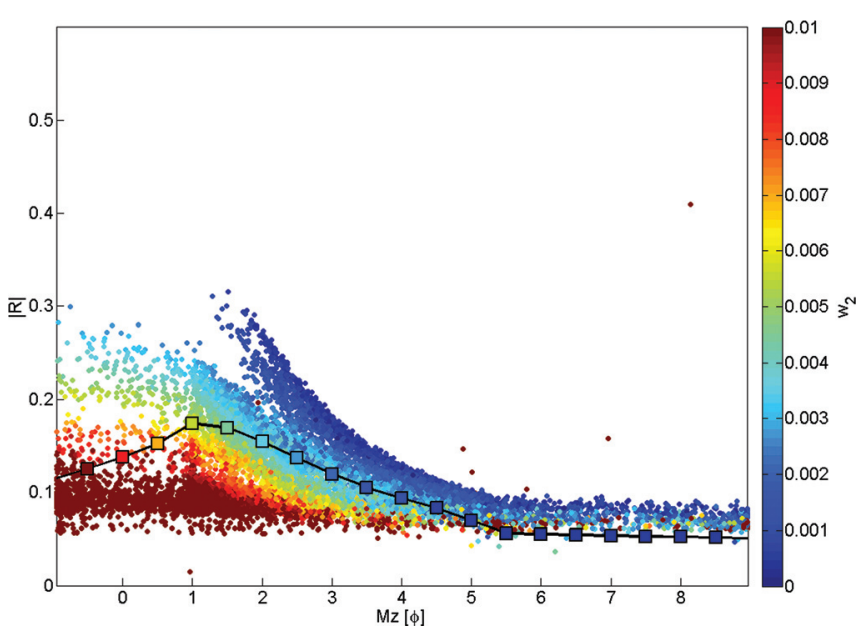

FIG. 12. The relation between the mean grain sizes inverted from the full echo envelope and the measured reflection coefficients (dots). The color of the dots represents the inverted spectral strength. For comparison, the graph of the simulated reflection coefficient is reproduced from Fig. 11. Here, the color of the squares represents the empirical value of the spectral strength. 
parameter, as obtained from the inversions, are higher than those predicted by the empirical relations for the higher $M_{z}$ values, resulting in increased signal energies and consequently increased estimates for the reflection coefficient, compared to that theoretically expected.

\section{CONCLUSIONS}

In this paper, two model-based approaches toward acoustic sediment classification with an SBES are investigated. The first bases the classification upon matching the complete echo envelope and inverting for the three parameters, mean grain size $M_{z}$, spectral strength $w_{2}$, and volume scattering parameter $\sigma_{2}$, whereas the second approach merely considers energy from the received signal to estimate $M_{z}$ only.

An application of the first approach to real data indicates its feasibility to estimate sediment parameters for a range of different sediment types. The estimates for the mean grain size are confirmed by the distribution of Folk classes, as determined from sediment samples taken in the area. No independent measurements are available for the spectral strength and volume scattering parameter. However, all three maps reveal a similar pattern of sediment distribution, as expected from the existence of empirical expressions relating these parameters. Plough marks in the area are clearly present in the map of the spectral strengths, and almost absent for the other two parameters, as expected. Whereas many applications mainly require information about the sediment mean grain sizes, information regarding the roughness and volume scattering parameters are of high relevance for application such as sonar performance prediction. The search for three parameters, however, makes this approach computationally demanding. Using a standard PC and MAT$\mathrm{LAB}$ as the programming language, real-time processing is not feasible, but on-line applications are expected to become feasible in the near future. A less computational demanding approach is to reduce the inverted parameters to $M_{z}$ only. This approach was found to still reveal a similar pattern for the sediment distribution. However, discrepancies between the inverted $M_{z}$ values and the values of $M_{z}$ from the grab samples are enlarged.

Classification based on a direct relation between echo energy and mean grain size, considered to eliminate the need for inversions completely, was found to be hampered by the limited beamwidth of the SBES. Coarse grained sediments were found to result in the same estimates for the mean grain size as finer grained sediments. The use of an SBES system with a larger beamwidth would allow for increased discriminating performance. Still, also the variation of the volume scattering parameter and the spectral strength around their empirical values prevents the coupling between echo energy and mean grain size; the unknown spectral strength and volume scattering parameter might then result in $M_{z}$ estimates that deviate from their true values.

Overall, it can be concluded that for model-based SBES classification, in practice, an approach based on the full echo envelope and accounting for $M_{z}, w_{2}$, and $\sigma_{2}$ is recommended. However, inverting for $M_{z}$ only or considering the echo energy, instead of using the full echo envelope, are approaches that are extremely useful for a first quick assessment of an area, on which for example decisions for further surveying can be based.

${ }^{1}$ C. de Moustier, "Beyond bathymetry: Mapping acoustic backscattering from the deep seafloor with Sea Beam," J. Acoust. Soc. Am. 79(2), 316331 (1986).

${ }^{2}$ J. E. Hughes Clarke, "Toward remote seafloor classification using the angular response of acoustic backscattering: A case study from multiple overlapping GLORIA data," IEEE J. Ocean. Eng. 19(1), 112-126 (1994).

${ }^{3}$ L. Hellequin, J. Boucher, and X. Lurton, "Processing of high-frequency multibeam echo sounder data for seafloor characterization," IEEE J. Ocean. Eng. 28(1), 78-89 (2003).

${ }^{4} \mathrm{G}$. Canepa and E. Pouliquen, "Inversion of geo-acoustic properties from high frequency multibeam data," in Boundary Influences in High Frequency, Shallow Water Acoustics, edited by N. G. Pace and P. Blondel (University of Bath Press, Bath, UK, 2005), pp. 233-240.

${ }^{5}$ D. G. Simons and M. Snellen, "A comparison between modeled and measured high frequency bottom backscattering," in Proceedings of the European Conference on Underwater Acoustics, Paris, France (June 29-July 4, 2008), pp. 639-644.

${ }^{6}$ D. G. Simons and M. Snellen, "A Bayesian approach to seafloor classification using multi-beam backscatter data," Appl. Acoust. 70, 1258-1268 (2009).

${ }^{7}$ A. R. Amiri-Simkooei, M. Snellen, and D. G. Simons, "Riverbed sediment classification using MBES backscatter data," J. Acoust. Soc. Am. 126(4), 1724-1738 (2009).

${ }^{8}$ W. Collins, R. Gregory, and J. Anderson, "A digital approach to seabed classification," Sea Technol. 37(8), 83-87 (1996).

${ }^{9}$ L. J. Hamilton, P. J. Mulhearn, and R. Poeckert, "Comparison of RoxAnn and QTC View acoustic bottom classification system performance for the Cairns area, Great Barrier Reef, Australia," Cont. Shelf Res. 19, 15771597 (1999).

${ }^{10} \mathrm{C}$. R. Bates and E. J. Whitehead, "ECHOplus measurements in Hopavågen bay, Norway," Sea Technol. 42(6), 34 (2001).

${ }^{11} \mathrm{C}$. Wienberg and A. Bartholomä, "Acoustic seabed classification in a coastal environment (outer Weser Estuary, German Bight)—A new approach to monitor dredging and dredge spoil disposal," Cont. Shelf Res. 25, 1143-1156 (2005).

${ }^{12}$ P. A. van Walree, J. Tegowski, C. Laban, and D. G. Simons, "Acoustic seafloor discrimination with echo shape parameters: A comparison with the ground truth," Cont. Shelf Res. 25, 2273-2293 (2005).

${ }^{13}$ D. D. Sternlicht and C. P. de Moustier, "Remote sensing of sediment characteristics by optimized echo-envelope matching," J. Acoust. Soc. Am. 114(5), 2727-2743 (2003).

${ }^{14}$ P. A. van Walree, M. A. Ainslie, and D. G. Simons, "Mean grain size mapping with single-beam echosounders," J. Acoust. Soc. Am. 120(5), 2555-2566 (2006).

${ }^{15} \mathrm{X}$. Lurton and E. Pouliquen, "Automated sea-bed classification system for echo-sounders," in Proceedings of the IEEE Oceans 1992 Conference (1992), pp. 317-321.

${ }^{16}$ D. D. Sternlicht and C. P. de Moustier, "Time-dependent seafloor acoustic backscatter (10-100 kHz)," J. Acoust. Soc. Am. 114(5), 2709-2725 (2003).

${ }^{17}$ K. V. Price, R. M. Storn, and J. A. Lampinen, Differential Evolution. A Practical Approach to Global Optimization (Springer, Berlin, 2005), Chap. 2.

${ }^{18}$ R. J. Urick, Principles of Underwater Sound, 3rd ed. (Peninsula Publishing, Los Altos, CA, 1983), Chap. 8.

19" APL-UW high-frequency ocean environmental acoustic models handbook," October 1994, Applied Physics Laboratory Technical Report APL-UW TR9407AEAS 9501.

${ }^{20} \mathrm{M}$. Snellen and D. G. Simons, "An assessment of the performance of global optimisation methods for geo-acoustic inversion," J. Comput. Acoust. 16(2), 199-223 (2008).

${ }^{21}$ D. G. Simons, K. Siemes, and M. Snellen, "The potential of inverting geo-technical and geo-acoustic sediment parameters from singlebeam echo sounder returns," in Proceedings of the Underwater Acoustic Measurements Conference, Nafplion, Greece (June 2009), pp. 163-170.

${ }^{22}$ D. G. Simons, M. Snellen, and M. A. Ainslie, "A multivariate correlation analysis of high frequency bottom backscattering strength measurements 
with geo-technical parameters," IEEE J. Ocean. Eng. 32(3), 640-650 (2007).

${ }^{23}$ R. L. Folk, "The distinction between grain size and mineral composition in sedimentary-rock nomenclature," J. Geol. 62(4), 344-359 (1954).
${ }^{24}$ M. Snellen, K. Siemes, and D. G. Simons, "On the practical applicability of single-beam model-based seafloor classification," in Proceedings of the European Conference on Underwater Acoustics, Istanbul, Turkey (July 59, 2010), pp. 274-278. 